\title{
Rectal ulcer in a hemodialysis patient receiving Sodium Polystyrene sulfonate ( Sodium KayexalateR)
}

\author{
Julia Collot ${ }^{1}$, Mohammed Salaouatchi ${ }^{2}$, Fabienne Rickaert ${ }^{3}$, Albino Floriani ${ }^{1}$, Benoit \\ Buysschaert $^{1}$, Maria Mesquita ${ }^{2}$, and Eric Godon ${ }^{1}$ \\ ${ }^{1}$ Centre Hospitalier Regional de Huy \\ ${ }^{2} \mathrm{CHU}$ Brugmann - Site Victor Horta \\ ${ }^{3} \mathrm{CHU}$ de Liège
}

December 30, 2020

\begin{abstract}

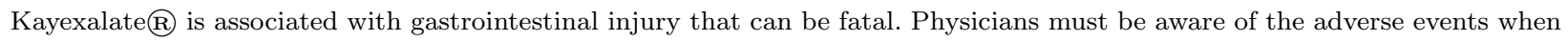
prescribing this therapy. Clinical data and medication history should follow the biopsy material given to the pathologist, who will look for Kayexalate@ crystals. Pathologists and physicians should work hand in hand.
\end{abstract}

Title page

Rectal ulcer in a hemodialysis patient receiving Sodium Polystyrene sulfonate (Kayexalateß)

Collot $\mathrm{J}^{1}$.

Nephrology and Dialysis Clinic, Centre Hospitalier Regional de Huy, Huy , Belgium

julia.collot@gmail.com

Salaouatchi Tayeb ${ }^{2}$

Nephrology and Dialysis Clinic, Internal Medicine Department Centre Hospitalier Universitaire, CHUBrugmann, Brussels, Belgium

salaouetchi.med.tayeb@gmail.com>

Fabienne Rickaert ${ }^{3}$

Department of Pathology, Centre Hospitalier Universitaire, Liège, Belgium

fabienne.rickaert@skynet.be>

Floriani $\mathrm{A}^{1}$.

Department of Gastroenterology ,Centre Hospitalier Regional de Huy , Huy , Belgium

albino.floriani@skynet.be

Buysschaert B ${ }^{1}$

Nephrology and Dialysis Clinic, Centre Hospitalier Regional de Huy , Huy , Belgium

benoit.buysschaert@chrh.be

Mesquita $\mathrm{M}^{2}$ 
Nephrology and Dialysis Clinic, Internal Medicine Department, Centre Hospitalier Universitaire, CHUBrugmann, Brussels, Belgium

maria.mesquita@ chu-brugmann.be

Godon $\mathrm{E}^{1}$.

Centre Hospitalier Regional de Huy , Huy , Belgium

eric.godon@chrh.be

Corresponding author :

Maria Mesquita

maria.mesquita@chu-brugmann.be

Abstract

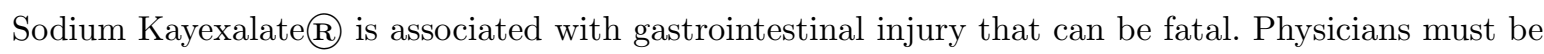
aware of the adverse events when prescribing this therapy. Clinical data and medication history

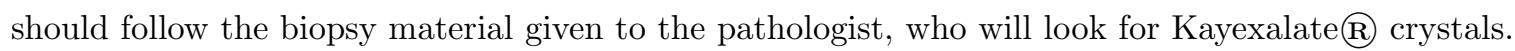
Pathologists and physicians should work hand in hand.

Key Words Hyperkaliemia, Hemodialysis, Rectal ulcer, Sodium Polystyrene Sulfonate (Sodium Kayexalate@), Kayexalate crystals.

Key Clinical message

Sodium Kayexalate $\AA$ can cause severe GI lesions. Diagnosis of Kayexalate crystals in GI biopsy samples is important. Pathologists and clinicians should work hand in hand. New drugs should be available to all patients to treat hyperkaliemia.

Introduction

Hyperkalemia may cause fatal arrhythmia in patients undergoing maintenance hemodialysis (HD) (1). In the majority of patients receiving HD, it is possible to avoid death from hyperkalemia with dietary therapy and adequate HD. However, some patients with oliguria or anuria receiving HD require treatment with an ionexchange resin such as sodium polystyrene sulfonate (SPS) (Sodium Kayexalate@) to prevent hyperkalemia.

Potassium plasmatic concentration is influenced by many other factors other than renal insufficiency such as Nonsteroidal anti-inflammatory drugs (NSAIDs), Angiotensin-converting enzyme (ACE) inhibitors and Angiotensin II Receptor Blockers (ARBs), glycaemia, acidosis, hemolysis and adrenal insufficiency which have also to be taken into account.

The use of SPS in the treatment of hyperkalemia may be associated with serious gastrointestinal (GI) adverse events. Side effects, with sometimes lethal sequelae, have been shown to occur in both the upper and lower GI tract in association with SPS use, and range from mild GI bleeding to perforation with acute abdomen (2).

The risk of these complications increases dramatically in patients with comorbidities such as kidney or vascular disease, patients with organ transplantation chronically treated with corticosteroids or immunosuppressive drugs and patients in the postoperative state. These patients are more susceptible to develop SPS induced GI injuries (3).

We report a case of circumferential ulcer of the rectum in a HD patient treated with Sodium Kayexalate $\mathbb{R}$. for chronic hyperkalemia. This patient had also several upper GI bleedings probably due to NSAIDs auto medication. 


\section{Case Report}

A 69 year old women on maintenance hemodialysis (HD) with a history of type 2 diabetes mellitus, hypertension, ischemic cardiomyopathy and chronic obstructive pulmonary disease (COPD), was treated by

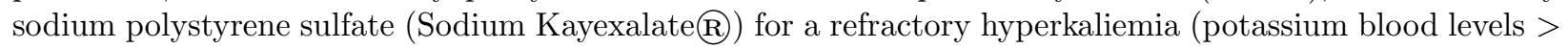
$6 \mathrm{mmol} / \mathrm{L}$ ) which was attributed to chronic abuse NSAIDs in the setting of end stage renal disease (ESRD).

The patient was also known for a history of two episodes of upper GI bleedings. First, on a large hemorrhagic duodenal ulcer and second on a serpiginous antral ulcer treated every time successfully with proton pump inhibitor (PPI).These ulcers were linked to NSAIDs consumption.

The patient presented hematochezia and the rectoscopy revealed a circumferential ulcer in the lower third of the rectum (Fig 1).

Histological examination of the rectal biopsies showed rectal mucosal ulcerations and rectangular basophilic crystals like "fish scales" adherent to the surface epithelium ( Fig 2) and colon mucosal ulceration with a crystal in the exudate. (Fig 3).

So, the diagnosis of Kayexalate $\AA$ - induced rectal injury was made on the basis of microscopic features, her clinical history and medication records. It is to be noted that she did not take any other medication resin such as sevelamer or bile duct sequestrants. Kayexalate $\mathbb{R}$ doses were first reduced, however it had to be continued for some time because of non-controlled hyperkaliemia.

For the time being, the patient is on HD thrice a week, she does not take any more NSAIDs, she pays attention to her diet and does not require anymore a potassium binder. Since 6 months the patient has no GI complaints and no digestive bleeding was observed.

\section{Discussion}

The GI tract is the target of several types of medical drugs including NSAIDs, iron pills, paclitaxel (Taxol@), Mycophenolate Mofetil (Cellcept@), bisphosphonates, colchicine and oral resins such as polystyrene sulfonate (SPS) and sevelamer used in renal failure patients. (4).

Sodium polystyrene sulfonate (SPS) is a potassium binding resin commonly used to treat mild acute or chronic hyperkaliemia by increasing the excretion of potassium in stool. It can be administrated orally or locally as an enema and it is sometimes associated with Sorbitol to prevent fecal impaction. It is a common drug prescribed in ESRD and hemodialysis patients (5).

It is known that the risk factors that contribute to GI injury associated with SPS use are chronic and end stage renal disease, solid organ transplantation patients treated with corticosteroids or immunosuppressive drugs and post-operative status. (3). Hence, our patient was at high risk to develop SPS related GI complications because she had ESRD. Nevertheless, this drug was prescribed because of uncontrolled hyperkaliemia and the risk of fatal cardiac arrhythmias. Our patient had persistent hyperkaliemia (blood levels $>6 \mathrm{mmol}$ /L) because of NSAIDs auto medication in the setting of ESRD and SPS was prescribed to maintain the potassium blood levels $<6 \mathrm{mmol} / \mathrm{L}$.

Kayexalate (B) was well tolerated and the patient did not have the common side effects of this drug such as loss of appetite, upset stomach, nausea, vomiting, constipation, or diarrhea (6).Moreover, it helped to reduce her potassium blood levels to $<6 \mathrm{mmol} / \mathrm{L}$. Studies have shown the tolerability and efficacy of SPS for long term management of hyperkalemia in patients chronic kidney disease (7). However, some authors doubt about the effectiveness and safety of this drug $(8-10)$.

In addition to the common side effects of SPS, mucosal ulceration including wall perforation and post inflammatory stricture formation are among the serious complications which may present as a clinical emergency. (11- 17).

Probably, if diagnosed early, a simple colonic ulcer can be diagnosed in some patients(18). Intestinal ischemia and necrosis has also been reported (19). Rectal ulcers and rectal stenosis can be caused by foreign body 
reaction to SPS crystals (20) (21).

In fact, review of the literature shows that this resin can induce not only intestinal, colonic and rectal ulcers, necrosis and perforations but that the upper gastrointestinal tract can also be injured because of this drug. (11) (22).

Our patient had a circumferential ulcer in the lower third of the rectum, a complication related to the use of SPS as shown at the histopathological examination of the biopsy.

Some authors describe colonic damage with the administration of SPS in sorbitol. SPS in sorbitol has been implicated in the development of intestinal necrosis, primarily mediated by the sorbitol component, leading to significant morbidity and mortality and the incidence of SPS-mediated intestinal injury has been estimated as $0.27 \%$ to $1.8 \%(19)(22)$.

Incidence of gastro-intestinal tract injuries because of sorbitol free SPS in CKD and dialysis patients is not known but seems very low. Nevertheless, SPS-induced intestinal ischemia remains an under recognized, easily avoidable complication, associated with significant morbidity and mortality. Physicians who routinely use this agent in sorbitol or without sorbitol should be aware of its life-threatening complications. As soon as the diagnosis is done the drug should be stopped in order to avoid further fatal complications. Sometimes clinicians are obliged to continue prescribing this drug because of lack of alternating drug for the moment.

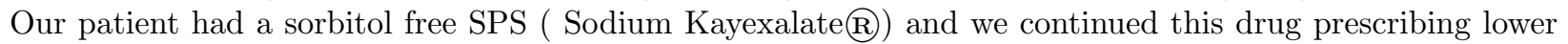
doses because of non-controlled hyperkaliemia that could lead to fatal cardiac arrhythmias.

The incidence of gastrointestinal injuries related to SPS (with or without sorbitol) varies in different population studies and according to the dose prescribed.

A retrospective cohort study based on the Swedish Renal Registry (SRR) showed that SPS is frequently used by persons with advanced CKD/ESKD chronically and at lower dosages than specified on the product label. Compared with non-use, they observed an increased relative risk of the combined endpoint of intestinal ischemia, thrombosis or ulceration/perforation for SPS use. They observed a dose dependent increase incidence of serious GI adverse events for CKD patients initiating SPS therapy. In addition, SPS use was associated with a higher rate of laxative dispensations. These associations were more evident in patients prescribed per label SPS dosages. (23).

Another study in older adults initiating SPS showed higher incidence of hospitalizations for serious GI adverse events among SPS users compared with non-users (24).

Sodium polystyrene sulfonate (SPS) is commonly prescribed for the treatment of hyperkalemia, however , two new potassium - binding agents, sodium zirconium cyclosilicate (ZS-9) and patiromer ( Veltassa), appear to have greater binding selectivity for potassium compared with that of SPS, but they are not yet available in all countries and for the treatment of all causes of hyperkaliemia (25-27).

The most common medication resins utilized in renal patients are sevelamer and kayexalate that chelate phosphate and potassium respectively. Both are associated with mucosal injury. Lesions seen in resin induced gastro-intestinal damage may overlap with those seen in inflammatory bowel disease, ischemic colitis, infectious colitis as well as microscopic colitis. The distinctive feature of resin mucosal damage is the identification of resin crystals. However, this was reported by the pathologist roughly in $75 \%$ of the cases (28).

The classic morphology to diagnose these crystals is as follows: Kayexalate is rectangular in shape, purple on hematoxylin- eosin staining (H\&E) and has "fish scales" or internal demarcations that appear similar to

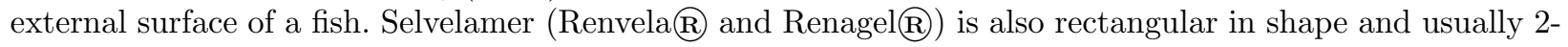
toned on H\&E, with pink center and yellow edges; it also has fish scales (28).

Classic morphology is the benchmark for identifying the resins, but color, location and fish scale pattern can deviate from the norm, making proper identification a challenge. Sometimes ancillary tools are required to distinguish them. Acid-fast bacillus special stain is the most reliable ancillary tool; it stains Kayexalate in 
black and Sevelamer in magenta. Discussion with the clinician about the medication taken by the patient is useful. It is critical for pathologists to be aware of the typical and atypical presentations of medication resins (28).

The typical histopathologic findings in SPS-induced necrosis include mucosal ulceration, necrosis and the presence of polygonal basophilic retractile crystals with a "fish scale" appearance. (3)(29). For pathologists, identifying the kayexalate crystals in necrotic gastrointestinal tissue is important, because early feedback to the clinician can lead to the cease of medication and avoidance of more serious adverse outcomes. Hence, clinicians and pathologist should work hand in hand.

Before she was taken for dialysis treatment because of ESRD, our patient presented antral and duodenal ulcers. Our pathologist took a second look in the gastric and duodenal biopsy samples in search once again

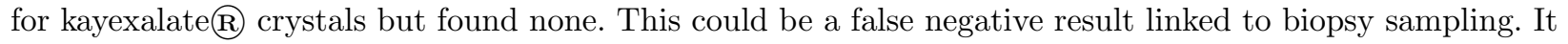
was suggested that these upper GI ulcers could be due to NSAIDs consumption.

\section{Conclusions}

Given the severity of GI complications, the therapeutic role of SPS needs to be re-evaluated. Although the risk to an individual patient may not be high, the wide spread use of this medication may be exposing a large population to potential risks, especially in high risk patients. As it is often given to fragile patients, it is important to use it cautiously to reduce as much as possible lethal side effects of this drug. Our observation suggests that physicians must be aware of the risk of these adverse events when prescribing SPS.

Alternative treatments in the management of hyperkaliemia in CKD / ESRD are the new armamentarium of potassium binders: patiromer and sodium zirconium cyclosilicate

These new drugs are not yet available in all countries. Moreover, randomized controlled studies should show the advantage of these new drugs in the protection of the GI tract in patients.

It is critical for pathologists to be aware of the typical and atypical presentations of medication resins, because early feedback to the clinician can lead to the cease of medication and avoidance of more serious adverse outcomes. Recognition and timely diagnosis can be life - saving. Hence clinicians and pathologist should work hand in hand.

Disclosures and conflict of interest : none

Financial support : none

Acknowledgements : the authors thank the hemodialysis nursing team.

Ethical approval : not applicable

Patient consent : Informed consent was obtained from the patient for the publication of this case report.

Author's contributions: CJ and MM have primary designed and drafted the paper.ST reviewed the related articles and also contributed in writing the manuscript, RF wrote the pathology content and obtained the images. FA, BB and GE revised the manuscript. All authors have read and approved the final version of the revised paper.

\section{References}

1. Yusuf A.A, Hu Y, Singh B. Menoyo J.A. Wetmore J.B. Serum Potassium Levels and Mortality in Hemodialysis Patients: A Retrospective Cohort Study. Am J Nephrol 2016;44:179-186

2. Harel Z , Harel S, Shah P.S, Wald R, Perl J, Bell C M . Gastrointestinal adverse events with sodium polystyrene sulfonate (Kayexalate) use: a systematic review. Am J Med . 2013;126 (3):264.e9-24.

3. Nuzzo A, Shaar-Chneker , Maillet M, Mégarbane B. Gastroduodenal injury induced by orally administered sodium polystyrene sulfonate Clin Toxicol (Phila) .2019; 57(1):75-76.

4. C.Panarelli N C. Drug-induced injury in the gastrointestinal tract Seminars in Diagnostic Pathology Volume 31, Issue 2, 2014, 165-175 
5. Nakamura T, Fujisaki T, Miyazono M , M. Yoshihara et al. Risks and Benefits of Sodium Polystyrene Sulfonate for Hyperkalemia in Patients on Maintenance Hemodialysis. Drugs R D . 2018;18(3):231-235..

6. Lepage L , Dufour A-C, Doiron J et al .Randomized Clinical Trial of Sodium Polystyrene Sulfonate for the Treatment of Mild Hyperkalemia in CKD , Clin J Am Soc Nephrol . 2015;10 (12):2136-42.

7. Panagiotis I, Liampas I, Kyriakou A.et al. Evaluation of the tolerability and efficacy of sodium polystyrene sulfonate for long-term management of hyperkalemia in patients with chronic kidney disease Int Urol Nephrol 2017 Dec; 49(12):2217-2221

8. Sterns R , Rojas M, Bernstein P, Chennupati S . Ion- exchange resins for the treatment of hyperkalemia: are they safe and effective? J Am Soc Nephrol .2010 ; 21(5):733-5

9. Malone D J. Taking a Second Look at Kayexalate. Hosp Pharm . 2015 ;50(11):959-60

10. Batterink J, Jane Lin , Hin Mui Au-Yeung S, Cessford T - Effectiveness of Sodium Polystyrene Sulfonate for Short-Term Treatment of Hyperkalemia. Can J Hosp Pharm . 2015; 68(4):296-303.

11. Abraham S C, B S Bhagavan, L A Lee, A Rashid, T T Wu . Upper gastrointestinal tract injury in patients receiving kayexalate (sodium polystyrene sulfonate) in sorbitol: clinical, endoscopic, and histopathologic findings. Am J Surg Pathol . 2001 May; 25(5):637-44

12. DardikA , Moesinger RC, G Efron G, A Barbul A, Harrison MG. Acute abdomen with colonic necrosis induced by Kayexalate-sorbitolSouth Med $J$. 2000 ;93(5):511-3

13. Piron L, Ramos J, Taourel P. Colonic perforation associated with sodium polystyrene sulfonate (Kayexalate) use. Diagn Interv Imaging . 2018; 99(7-8):507-509

14. Kao C-C, Tsai Y-C , Chiang W-C , Mao T-L , Kao T-W. Ileum and colon perforation following peritoneal dialysis-related peritonitis and high-dose calcium polystyrene sulfonate. J Formos Med Assoc . $2015 ; 114(10): 1008-10$.

15. Dunlap R H, Ryan Martinez Total colectomy for colon perforation after kayexalate administration: a case report and literature review of a rare complication . Case Reports J Surg Case Rep . 2016 :10;1-3

16. Chou Y-H, Wang H-Y, Hsieh M-S. Colonic necrosis in a young patient receiving oral kayexalate in sorbitol: case report and literature review. Kaohsiung J Med Sci . 2011; 27(4):155-8.

17. Joo M. , Bae WK, Kim N.H, Han S.R. Colonic mucosal necrosis following administration of calcium polystryrene sulfonate (Kalimate) in a uremic patient. J Korean Med Sci . 2009 ; 24(6):1207-1.

18. Albeldawi M , Gaur V, Weber L Kayexalate-induced colonic ulcer Gastroenterol Rep (Oxf) . 2014 ; $2(3): 235-6$.

19. McGowan C E , Saha S, Chu G, Resnick M B, Moss SF. Intestinal necrosis due to sodium polystyrene sulfonate (Kayexalate) in sorbitol. South Med J . 2009 ; 102 (5):493-7.

20. Chatelain D , Brevet M, Manaouil D, YzetnT, Regimbeau J-M, Sevestre H .Rectal stenosis caused by foreign body reaction to sodium polystyrene sulfonate crystals (Kayexalate). Ann Diagn Pathol2007;11(3):217-9.

21. Oliveira A.A , Pedro F , Craveiro N , A.V , Almeida R.S , Luís P.P , Santos C. Rectal ulcer due to Kayexalate deposition - an unusual case . Rev Assoc Med Bras 2018 ; 64 (8):680-683.

22. Rashid A , Hamilton S;R. Necrosis of the gastrointestinal tract in uremic patients as a result of sodium polystyrene sulfonate (Kayexalate) in sorbitol: an under recognized condition. Am J Surg Pathol . 1997; 21(1) : 60-9.

23. Laureati P, Xu Y , Trevisan M , Schalin L , Mariani I, Bellocco R, Sood M.M, Barany P , Sjölander A, Evans M , CarrerJ.J. Initiation of sodium polystyrene sulphonate and the risk of gastrointestinal adverse events in advanced chronic kidney disease: a nationwide study. Nephrol Dial Transplant . $2019 ; 1-9$

24. Noel J.A , Bota S.E , Petrcich W , GargA.X , Carrero J.J , Harel Z , Tangri N , ClarkE.G , Komenda P , Sood M.M Risk of Hospitalization for Serious Adverse Gastrointestinal Events Associated With Sodium Polystyrene Sulfonate Use in Patients of Advanced Age JAMA Intern Med . 2019 10;179 (8):1025-1033.

25. Chaitman M, Dixit D, Bridgeman M.B. Potassium-Binding Agents for the Clinical Management of Hyperkalemia $P \&$ T . 2016,41 (1):43-50.

26. Beccari M.V, Meaney C.J. Clinical utility of patiromer, sodium zirconium cyclosilicate, and sodium 
polystyrene sulfonate for the treatment of hyperkalemia: an evidence-based review. Core Evidence . 2017 23;12:11-24.

27. Meaney C.J, Beccari M.V, YangY, Zhao J. Systematic Review and Meta-Analysis of Patiromer and Sodium Zirconium Cyclosilicate: A New Armamentarium for the Treatment of Hyperkalemia. Pharmacotherapy $2017 ; 37(4): 401-411$.

28. Gonzalez R.S, Lagana S.M, Szeto O, Arnold C.A Challenges in Diagnosing Medication Resins in Surgical Pathology Specimens: A Crystal-Clear Review Guide. Arch Pathol Lab Med . 2017 ;141(9):12761282.

29. Bogaerts J.M.A 'Van der Hoeven J.G , Arts E.E.A , Van der Kolk BM , Brosens LA . Fish scale crystals: an under-recognized cause of intestinal necrosis. J Clin Pathol . 2019 ;72(8):567

\section{Legends :}

- Image 001: circumferential ulcer in the lower third of the rectum

- Image 002: refractile basophilic crystal with typical fish scales morphology

- Image 003 : colon mucosal ulceration with a crystal in the exudate

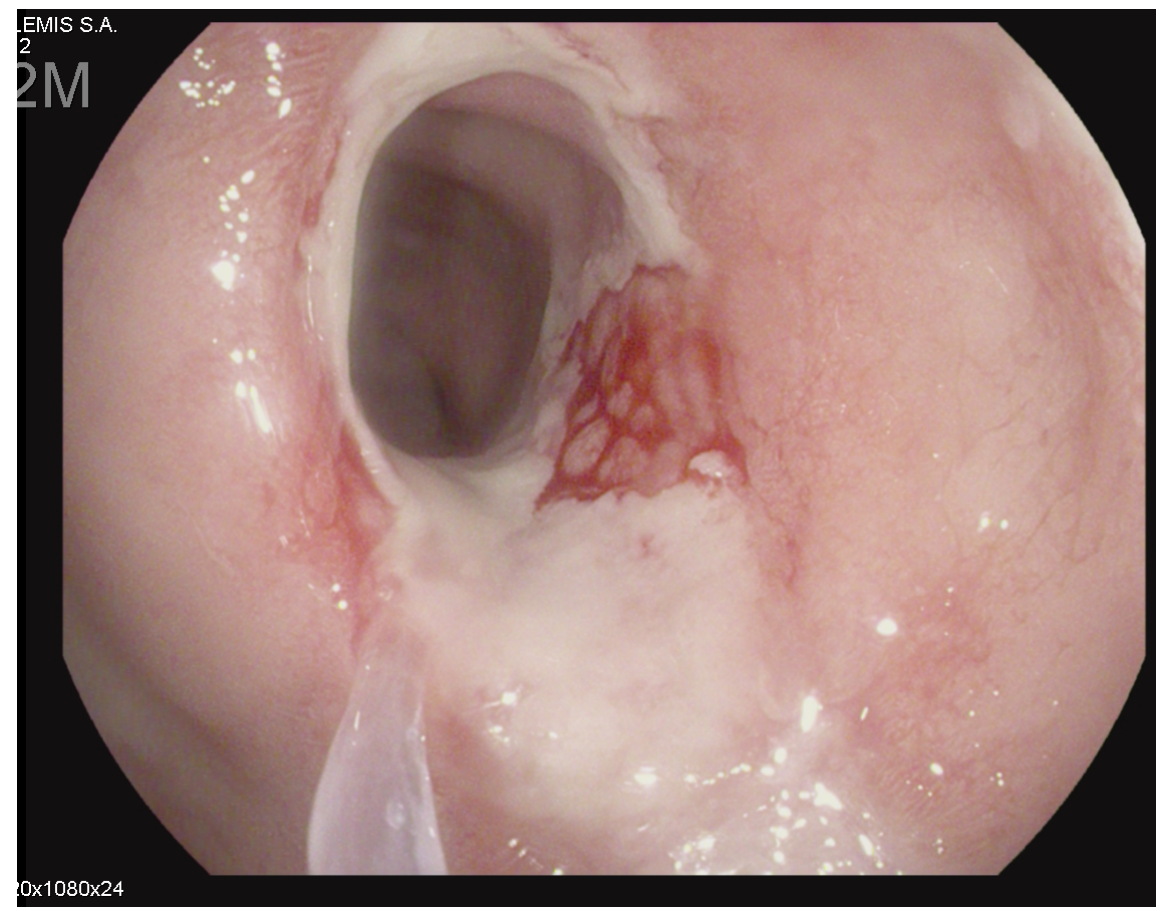



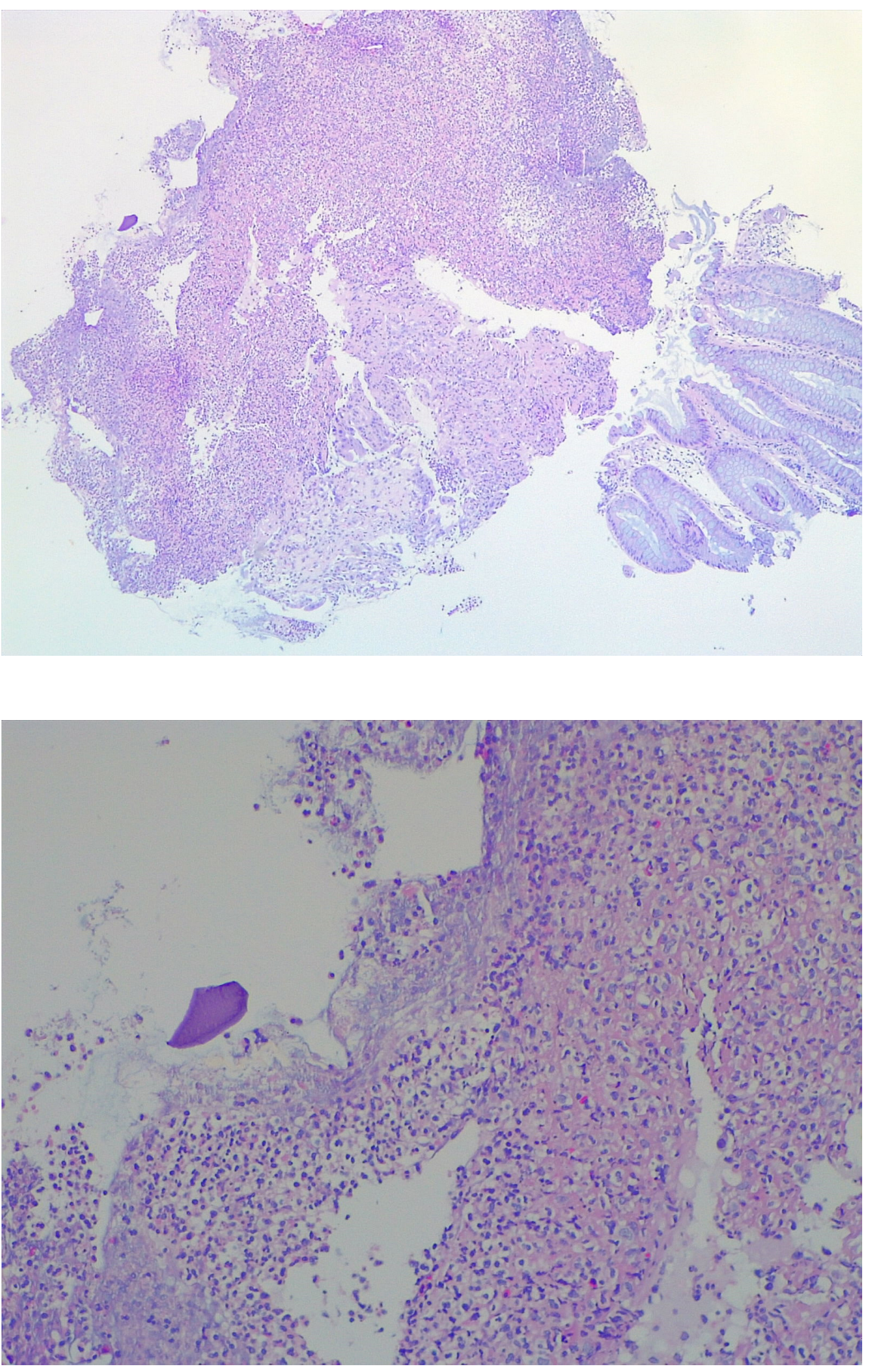